\title{
High-Energy Neutrinos from NGC 1068
}

\author{
Luis A. Anchordoqui, ${ }^{a, b, c, *}$ John F. Krizmanic ${ }^{d, e}$ and Floyd W. Stecker ${ }^{d, f}$ \\ ${ }^{a}$ Department of Physics, Lehman College, City University of New York, NY 10468, USA \\ ${ }^{b}$ Department of Physics, Graduate Center, City University of New York, NY 10016, USA \\ ${ }^{c}$ Department of Astrophysics, American Museum of Natural History, NY 10024, USA \\ ${ }^{d}$ NASA/Goddard Space Flight Center, Greenbelt, MD 20771, USA \\ ${ }^{e}$ Department of Physics, University of Maryland, Baltimore, MD 21250, USA \\ ${ }^{f}$ Department of Physics and Astronomy, University of California, Los Angeles, CA 90095, USA
}

IceCube has observed an excess of neutrino events over expectations from the isotropic background from the direction of NGC 1068. The excess is inconsistent with background expectations at the level of $2.9 \sigma$ after accounting for statistical trials. Even though the excess is not statistical significant yet, it is interesting to entertain the possibility that it corresponds to a real signal. Assuming a single power-law spectrum, the IceCube Collaboration has reported a best-fit flux $\phi_{v} \sim 3 \times 10^{-8}\left(E_{v} / \mathrm{TeV}\right)^{-3.2}\left(\mathrm{GeV} \mathrm{cm}^{2} \mathrm{~s}\right)^{-1}$, where $E_{v}$ is the neutrino energy. Taking account of new physics and astronomy developments we give a revised high-energy neutrino flux for the Stecker-Done-Salamon-Sommers AGN core model and show that it can accommodate IceCube observations.

$37^{\text {th }}$ International Cosmic Ray Conference (ICRC 2021)

July 12 th $-23 r d, 2021$

Online - Berlin, Germany

\footnotetext{
*Presenter
} 
A search for astrophysical point-like neutrino sources using $10 \mathrm{yr}$ of data collected by the IceCube detector (between April 6, 2006 and July 10, 2018) finds an excess of clustered events (with energies $E_{v} \gtrsim 1 \mathrm{TeV}$ ) over expectations from an isotropic sky coincident with the Seyfert II galaxy NGC 1068 [1]. The excess is inconsistent with background expectations at the level of $2.9 \sigma$ after accounting for statistical trials. When the distributions of the observed events as a function of their distance from NGC 1068 and their estimated angular uncertainties are weighted by a signal-over-background likelihood characterizing the point-like source hypothesis give a best fit spectrum $\propto E_{v}^{-3.2}$. On the assumption of a single power-law spectrum IceCube finds a best-fit flux $\phi_{v} \sim 3 \times 10^{-8}\left(E_{v} / \mathrm{TeV}\right)^{-3.2}\left(\mathrm{GeV} \mathrm{cm}^{2} \mathrm{~s}\right)^{-1}$; the reconstructed muon neutrino spectrum with its large uncertainty is shown in Fig. 1. A point worth noting at this juncture is that the favored soft spectrum for NGC 1068 is consistent with the shape of the high-energy starting event all-sky neutrino spectrum, which is compatible with an unbroken power-law spectrum, with a preferred spectral index of $2.87_{-0.19}^{+0.20}$ for the $68.3 \%$ confidence interval [5].

Recently, the MAGIC Collaboration reported a search for gamma-ray emission in the veryhigh-energy band [4]. No significant signal was detected during 125 hours of observation of NGC 1068. The null result provides a 95\% CL upper limit to the gamma-ray flux above $200 \mathrm{GeV}$ of $5.1 \times 10^{-13}\left(\mathrm{~cm}^{2} \mathrm{~s}\right)^{-1}$. This limit improves an earlier upper bound from H.E.S.S. [3] and set tight constraints on the theoretical models that could explain the NGC 1068 IceCube's "signal." More concretely, the gamma rays accompanying the neutrino flux must be significantly attenuated; see Fig. 1. The gamma-ray optical depth is well-known,

$$
\tau_{\gamma \gamma}(\varepsilon) \sim \frac{\sigma_{\gamma \gamma}}{4 \pi c} \frac{L_{X}}{\varepsilon R} \sim 10^{5}\left(\frac{\varepsilon}{1 \mathrm{keV}}\right)^{-1}\left(\frac{L_{X}}{L_{\mathrm{Edd}}}\right)\left(\frac{R_{S}}{R}\right),
$$

where $\varepsilon$ is the typical energy of the target photon background, $\sigma_{\gamma \gamma}$ is the scattering cross section, $L_{X}$ is the $X$-ray luminosity, $R$ is the size of the region carrying the dense $X$-ray target photons, $R_{S}=$ $2 G M / c^{2}$ is the is the Schwarzschild radius, $L_{\mathrm{Edd}}=4 \pi G M m_{p} c / \sigma_{T}$ is the Eddington luminosity (i.e., the maximum steady state luminosity that can be produced before radiation pressure disrupts the accretion flow), and $\sigma_{T}$ is the Thomson cross section, with $M$ and $m_{p}$ the black hole and proton mass, respectively. It is straightforward to see using (1) that to have significant absorption of the gamma rays $R$ must characterize a compact region in the vicinity of the black hole.

In 1991, Stecker, Done, Salamon, and Sommers proposed a model featuring all of these characteristics [6]. The high-energy neutrino flux originates in the core of the active galactic nucleus (AGN). Protons can reach very high energies through accretion-disk shock-acceleration at the inner edge of the black hole [7]. The relativistic protons undergo inelastic collisions with the thermal photon background to produce charged and neutral pions, which, in turn, decay into neutrinos, electrons, and gamma-rays. The non-thermal electrons generate gamma rays via inverse Compton scattering on disk photons. While all the gamma rays cascade down to the $\mathrm{MeV}$ energy range because of the strong internal attenuation effect [8] neutrinos escape the source en route to Earth. Taking account of new physics and astronomy developments, in this communication we give a revised high energy neutrino flux for the AGN core model and show that it can accommodate IceCube data. Observational studies and theoretical modeling are used to guide us in choosing the model parameters. Before proceeding, we pause to note that related ideas for modelling neutrino emission from NGC 1068 have been discussed in [9-12]. 


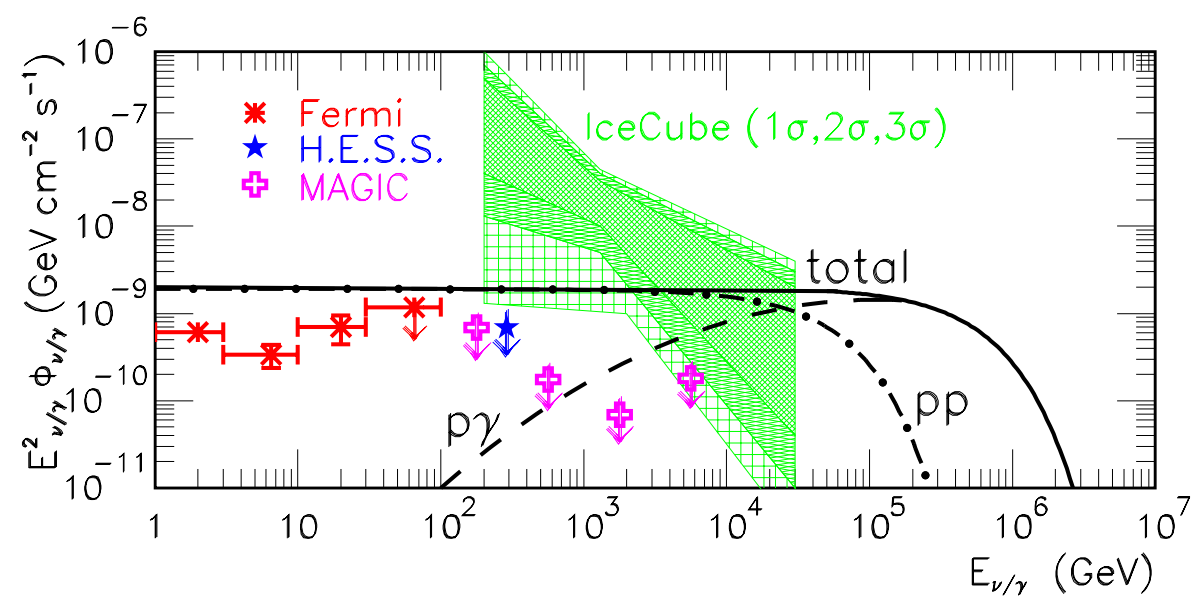

Figure 1: AGN-core model prediction of the muon neutrino spectrum from NGC 1068; $p p$ interactions (dot-dashed line), $p \gamma$ interactions (dashed line), and total (solid line). For comparison, we overplot the best-fit time-integrated astrophysical power-law neutrino flux obtained using the $10 \mathrm{yr}$ IceCube data [1]. We also show measurements and bounds on the gamma-ray flux from Fermi [2], H.E.S.S. [3] and MAGIC [4]. We have set $b=1$ in our calculations.

$X$-ray absorbers are classified as Compton-thick or -thin, according to whether their column density $N_{\mathrm{H}}$ is larger or smaller than $\sigma_{T}^{-1} \simeq 1.5 \times 10^{24} \mathrm{~cm}^{-2}$. Back in the $90 \mathrm{~s}$, there was a lack of evidence for strong $X$-ray absorption features in AGN spectra [13, 14], and this was taken as an indication that the secondary $X$-rays are produced in regions of low column density. If this were the case, the amount of target gas for $p p$ collisions would be very limited and the very large photon density in the AGN core would make photopion production, predominantly through the resonant process $p \gamma \rightarrow \Delta^{+} \rightarrow n \pi^{+}$or $p \pi^{0}$, the leading mechanism for energy loss. Because of resonant scattering the mean pion energy is kinematically determined by requiring equal boosts for the decay products of the $\Delta^{+}$, giving $\left\langle E_{\pi}\right\rangle \sim E_{p} / 5$ [15]. Likewise, to a first approximation we relate the energy of the neutrinos with that of the parent protons considering that the four (massless) particles resulting from the decay $\pi^{+} \rightarrow \mu^{+} v_{\mu} \rightarrow e^{+} v_{e} \bar{v}_{\mu} v_{\mu}$ (and the charge-conjugate processes) share similar amounts of energy $\left\langle E_{v}\right\rangle \simeq\left\langle E_{\pi}\right\rangle / 4 \simeq E_{p} / 20$. For the neutral pions $\pi^{0} \rightarrow \gamma \gamma$, we similarly find $\left\langle E_{\gamma}\right\rangle \simeq E_{p} / 10$. The threshold condition for pion production in $p \gamma$ scattering is given by $\left(p_{\gamma}+p_{p}\right)^{2}>\left(m_{p}+m_{\pi}\right)^{2}$, which leads to $\zeta>\left(2 m_{\pi} m_{p}+m_{\pi}^{2}\right) / m_{p}^{2} \equiv \zeta_{0} \simeq 0.313$, where the dimensionless variable $\zeta \equiv 4 \varepsilon E_{p} / m_{p}^{2}$ characterizes the center-of-mass total energy squared of the interaction and where we have taken $m_{\pi}^{ \pm} \simeq m_{\pi}^{0} \simeq 137 \mathrm{MeV}$ and $m_{p} \simeq m_{n} \simeq 938 \mathrm{MeV}$. For UV photons, with a mean energy $\langle\varepsilon\rangle \sim 40 \mathrm{eV}$, this translates into a characteristic proton energy $E_{p, \text { min }}>70 \mathrm{PeV} /(\varepsilon / \mathrm{eV}) \sim 2 \mathrm{PeV}[6]$. The fact that this reaction turns on at so high energies implies that the photons and neutrinos from decaying pions are produced at very high energies too, well above the $\mathrm{TeV}$ range. Thereby, IceCube's observation of $O(\mathrm{TeV})$ neutrinos from the direction of NGC 1068 pose unique challenges for predictive modeling.

Over the past decades, multiple space-missions and ground-based experiments (including BeppoSAX, Chandra, MERLIN, the Very Long Baseline Array, NuSTAR, and XMM-Newton [1621]) have performed an extensive observing campaign aimed at the characterization of NGC 1068. 
Collectively, these observations call for a recalibration of the AGN-core-model parameters. In particular, NuSTAR detected a flux excess above $20 \mathrm{keV}$ with respect to both the December 2012 observation and a later observation performed in February 2015. The most plausible explanation of the NuSTAR transient excess is that for a short time interval the total absorbing column, probably composed by a number of individual clouds, became less thick so as to allow the radiation from the AGN core to pierce through it, supporting the hypothesis of a clumpy structure of the obscuring material along the line of sight. The inferred column gas density from NuSTAR observations, which varies in the range $5.9 \times 10^{24} \lesssim N_{\mathrm{H}} / \mathrm{cm}^{2} \lesssim 8.5 \times 10^{24}$ [21], seems to indicate that the target proton gas in the AGN core is much denser than previously thought [14]. If this were the case, NGC 1068 should be reclassified as an optically thick absorber. For $p p$ collisions, threshold effects are insignificant and so for column densities $N_{H}>\sigma_{T}^{-1}, p p$ scattering could produce a TeV neutrino population to explain the low-energy tail of IceCube's "signal" [1]. Moreover, after correction for absorption, the inferred intrinsic $X$-ray luminosity of NGC 1068 (in the $2-10 \mathrm{keV}$ range) is $L_{X}=6_{-4}^{+7} \times 10^{43} \mathrm{erg} \mathrm{s}^{-1}$ [21], above about 2 orders of magnitude than previous estimates [17]. It is important to note that the intrinsic $L_{X}$ of NGC 1068 is roughly an order of magnitude larger than the $L_{X}$ of NGC 4151 [22,23], which is the brightest Seyfert in $X$-rays. Since both these two sources are located at about $14 \mathrm{Mpc}$ from Earth [24], the $L_{X}$ recalibration of [21] makes NGC 1068 the intrinsically brightest Seyfert galaxy in the sky, and explains why it could become the first neutrino source to be uncovered using (only) IceCube data. ${ }^{1}$

To develop some sense for the orders of magnitude involved, we begin by noting that firstorder Fermi acceleration of protons in strong (non-relativistic) shocks produces a power-law proton energy spectrum $\propto E_{p}^{-2}$ up to a maximum energy $E_{p, \max }$. The proton acceleration time-scale is given by

$$
t_{\mathrm{acc}}\left(E_{p}\right) \sim 5 \times 10^{-2} b\left(\frac{R_{\text {shock }}}{R_{S}}\right)\left(\frac{B}{\mathrm{G}}\right)^{-1}\left(\frac{E_{p}}{m_{p}}\right) \mathrm{s},
$$

where $B \simeq 5.5 \times 10^{27} Q^{-1 / 2}\left(R_{\text {shock }} / R_{S}\right)^{-7 / 4} L_{X}^{-1 / 2} \mathrm{G}$ is the magnetic field, $R_{\text {shock }}$ is the shock radius, $Q=1-0.1\left(R_{\text {shock }} / R_{S}\right)^{0.31}$ is the efficiency of conversion of bulk kinetic energy of accreting plasma into energetic particles at the shock, and $b$ is a numerical factor that gives a measure of the particle's mean free path (in gyroradii) for scattering off the magnetic field inhomogeneities [26, 27]. Based on the assumption $L_{X} \sim L_{\mathrm{Edd}} / 20$ (which corresponds to $M \sim 10^{7} M_{\odot}$ ) we fix the shock radius to $R_{\text {shock }} \sim 10 R_{S}[6]$.

The $p p$ energy-loss rate is given by

$$
t_{p p}\left(E_{p}\right)=\frac{1}{n_{p} \sigma_{p p} c \kappa_{p p}}
$$

where $\sigma_{p p}\left(E_{p}\right) \sim\left[34.3+1.88 \ln \left(E_{p} / \mathrm{TeV}\right)+0.25 \ln ^{2}\left(E_{p} / \mathrm{TeV}\right)\right] \times 10^{-26} \mathrm{~cm}^{2}$ is the inelastic $p p$ cross section [28], $\kappa_{p p} \sim 0.5$ is the proton inelasticity of the process [29], and $n_{p} \sim N_{\mathrm{H}} / R$ the mean proton density. Following [6], we take $R \sim 30 R_{S}$.

\footnotetext{
${ }^{1}$ Multimessenger observations of TXS 0506+056 provided $3 \sigma$ evidence of neutrino emission from the flaring blazar [25]. However, the association of the Texas source with neutrino emission in IceCube's $10 \mathrm{yr}$ data sample is less significant [1] than the reported significance of the time-dependent flare associating both neutrino and gamma-ray production.
} 
The $p \gamma$ energy-loss rate is evaluated by

$$
t_{p \gamma}^{-1}\left(E_{p}\right)=\frac{c}{2} \int_{0}^{\infty} d \varepsilon \frac{n(\varepsilon)}{\gamma^{2} \varepsilon^{2}} \int_{0}^{2 \gamma \varepsilon} d \varepsilon^{\prime} \varepsilon^{\prime} \kappa_{p \gamma} \sigma_{p \gamma}\left(\varepsilon^{\prime}\right),
$$

where $\gamma=E_{p} / m_{p} c^{2}$ is the Lorentz boost, $\varepsilon^{\prime}$ is the photon energy in the proton rest frame, $n(\varepsilon)$ is the differential number density of photons, and $\sigma_{p \gamma}$ and $\kappa_{p \gamma}$ are the cross section and inelasticity for photopion production, respectively [30]. We approximate the $p \gamma$ cross section by interactions with the $\Delta^{+}$resonance of mass $m_{\Delta} \simeq 1.232 \mathrm{GeV}$. Since the decay width $\Gamma_{\Delta} \simeq 150 \mathrm{MeV}$ is much smaller than the resonance mass the cross section can be safely approximated by the single pole of the narrow-width approximation,

$$
\sigma_{p \gamma}\left(\varepsilon^{\prime}\right)=\pi \sigma_{0} \frac{\Gamma}{2} \delta\left(\varepsilon^{\prime}-\varepsilon_{0}\right)
$$

where $\sigma_{0} \simeq 5 \times 10^{-28} \mathrm{~cm}^{2}$ is the resonance peak and $\varepsilon_{0}=\left(m_{\Delta}^{2}-m_{p}^{2}\right) /\left(2 m_{p}\right) \simeq 340 \mathrm{MeV}$ the pole. The factor of $\pi / 2$ is introduced to match the integral (i.e. total cross section) of the Breit-Wigner and the delta function. The photopion cooling rate can now be readily obtained substituting (5) into (4),

$$
\begin{aligned}
t_{p \gamma}^{-1}\left(E_{p}\right) & \approx \frac{c \pi \sigma_{0} \varepsilon_{0} \Gamma_{\Delta} \kappa_{p \gamma}}{4 \gamma^{2}} \int_{0}^{\infty} \frac{d \varepsilon}{\varepsilon^{2}} n(\varepsilon) \Theta\left(2 \gamma \varepsilon-\varepsilon_{0}\right)=\frac{c \pi \sigma_{0} \varepsilon_{0} \Gamma_{\Delta} \kappa_{p \gamma}}{4 \gamma^{2}} \int_{\epsilon_{0} / 2 \gamma}^{\infty} \frac{d \varepsilon}{\varepsilon^{2}} n(\varepsilon) \\
& =\frac{c \pi \sigma_{0}\left(m_{\Delta}^{2}-m_{p}^{2}\right) \Gamma_{\Delta} \kappa_{p \gamma}}{8 m_{p}}\left(\frac{m_{p}}{E_{p}}\right)^{2} \int_{\varepsilon_{\min }}^{\infty} \frac{d \varepsilon}{\varepsilon^{2}} n_{\gamma}(\varepsilon)
\end{aligned}
$$

where $\varepsilon_{\min }=\left(m_{\Delta}^{2}-m_{p}^{2}\right) /\left(4 E_{p}\right)$ [31]. We assume that the spectrum of the external UV radiation field arises from a Shakura-Sunyaev optically-thick accretion disk model that is scattered by clouds [32]. For calculations, we approximate the AGN continuum $n(\varepsilon)$ by two components: (i) a power-law spectrum $\propto \varepsilon^{-1.7}$ which extends up to $1 \mathrm{MeV}$ and (ii) a black body spectrum with temperature $T=5 \times 10^{4} \mathrm{~K}$ used to represent the UV/optical bump which is thought to be thermal emission from the accretion disk [27]. For normalization, we assume that the total $X$-ray luminosity is roughly the same as that in the UV-bump $L_{X} \sim L_{U V}$ and so $L_{C} \sim 4 \pi R^{2} c \int \varepsilon n(\varepsilon) d \varepsilon=L_{\mathrm{Edd}} / 10$, where $L_{C}$ is the luminosity in the infrared to hard $X$-ray continuum [6].

Now, by equating (2) to (3)+ (6) with $b=1$ it is easily seen that $E_{p \text {, max }}$ is $O\left(10^{7} \mathrm{GeV}\right)$. The order of magnitude estimate from this back-of-the-envelope calculation is consistent with the result from a Monte Carlo simulation, which gives

$$
E_{p, \max } \simeq 1.8 \times 10^{7} \mathrm{GeV}\left(6 / b^{2}\right)^{\alpha},
$$

where $\alpha=0.52$ for $b^{2}<6$ and $\alpha=0.18$ for $b^{2}>6$ [33]. Armed with (2), (3), (6), and (7), together with the inclusive pion spectra and the energy spectra of photons and leptons produced at $p p[28,34]$ and $p \gamma[34,35]$ collisions it is straightforward to calculate the muon neutrino yield from NGC 1068. Our results are encapsulated in Fig. 1. At low energies the spectrum $\propto E_{v}^{-2}$ from $p p$ interactions dominates; at high energies the spectrum from $p \gamma$ interactions dominates. Corrections due to kaon decay and threshold effects are $O(10 \%)$ [36] and fall within erros. We have accounted for a reduction in the muon-neutrino flux at production by a factor of 2 due to neutrino 
oscillations (whose discovery was made after the publication of [6]). From (1) we can see that the accompanying photons from $\pi^{0}$ decay cascade down to lower energies, in agreement with the upper limits from H.E.S.S. [3] and MAGIC [4].

We now turn to compare our results with recent estimates of the neutrino flux from NGC 1068. The predicted neutrino flux is in agreement with the estimates of [9-11]. However, it is important to stress that the acceleration rate adopted in our study is significantly faster than the one used in [911]. This implies that the maximum energy is always controlled by $p \gamma$ interactions. In particular, $t_{p \gamma}\left(E_{p, \text { max }}\right) \ll t_{p p}\left(E_{p, \max }\right)$ even when considering the upper bound of $n_{p} \sim 9 \times 10^{10} \mathrm{~cm}^{-3}$. For the acceleration mechanisms entertain in [9-11], the column density cannot (significantly) surpass $\sigma_{T}^{-1}$ otherwise $p p$ collisions would control and largely reduce $E_{p \text {, max }}$. The neutrino flux predicted by the AGN-core model is about an order of magnitude larger than the estimate in [12], which is normalized to accommodate gamma-ray observations.

Although there are a few other nearby AGN of this magnitude which can potentially be detected as point sources, one can integrate over the estimated AGN population out to the horizon to obtain a prediction for the diffuse neutrino flux. The result is simple: $\Phi_{v} \sim \frac{1}{4 \pi} \mathcal{R} n_{\mathrm{AGN}}\left\langle L_{v}\right\rangle$, where $\mathcal{R} \simeq 1$ horizon $\simeq 3 \mathrm{Gpc}, n_{\mathrm{AGN}} \sim 800 \mathrm{Gpc}^{-3}$ is the number density of AGN with $L_{X}>$ $10^{43} \mathrm{erg} / \mathrm{s}$ [37], and $\left\langle L_{v}\right\rangle$ is an average AGN neutrino luminosity (all flavors). What has become of the energy red-shifting of the neutrino? A more careful calculation must include an additional factor, $H_{0} \int d z H^{-1}(z) L_{v}(z) / L_{v}(0)$, to account for effects of the expanding universe (viz., loss of energy associated with the redshift $z$ and also depending on a choice of Hubble parameter $H$ ) and possible source evolution $[6,38,39]$. However, given the large uncertainty in the energy spectrum, we will ignore this order of magnitude "correction" and just note that if $\left\langle L_{v}\right\rangle \sim 10^{-2} L_{X} E_{v}^{-2}$, the diffuse neutrino flux expected on Earth from the AGN population, $E_{v}^{2} \Phi_{v} \sim 10^{-8} \mathrm{GeV}\left(\mathrm{cm}^{2} \mathrm{sr} \mathrm{s}\right)^{-1}$, would be in the ballpark of IceCube observations [5]. Curiously though, there is a seemingly bumpy-structure in the spectrum of the high-energy starting event sample around the $100 \mathrm{TeV}$ energy bin. Coincidentally, this is the energy range in which photopion production on the disk photons turns on. It is then tempting to speculate that if not all AGN are Compton-thick we would expect a bump in the spectrum when AGN sources producing neutrinos only via $p \gamma$ interactions come into play.

In summary, IceCube has detected an intriguing excess of events above the isotropic background from the direction of NGC 1068. We have shown that the origin of these neutrinos can be traced back to a Fermi engine at the core of this AGN. Absorption and interactions intrinsic to the source due to the high opacity, will result in a suppressed TeV gamma-ray flux to accommodate H.E.S.S. and MAGIC upper limits. The neutrino AGN-core model is fully predictive and will be confronted with future IceCube data.

\section{Acknowledgments}

LAA is supported by NSF Grant PHY-1620661 and NASA Grant 80NSSC18K0464. JFK is supported by NASA Grant 80NSSC19K0626. FWS is suppoorted by NASA Fermi Grant 80NSSSC20K0413. 


\section{References}

[1] M. G. Aartsen et al. [IceCube Collaboration], Phys. Rev. Lett. 124, 051103 (2020) [arXiv: 1910.08488].

[2] A. Lamastra, F. Fiore, D. Guetta, L. A. Antonelli, S. Colafrancesco, N. Menci, S. Puccetti, A. Stamerra and L. Zappacosta, Astron. Astrophys. 596, A68 (2016) [arXiv:1609.09664].

[3] F. Aharonian et al. [H.E.S.S. Collaboration], Astron. Astrophys. 441, 465 (2005) [arXiv:astro$\mathrm{ph} / 0507207]$.

[4] V. A. Acciari et al. [MAGIC Collaboration], Astrophys. J. 883, 135 (2019) [arXiv:1906.10954].

[5] R. Abbasi et al. [IceCube Collaboration], [arXiv:2011.03545].

[6] F. W. Stecker, C. Done, M. H. Salamon and P. Sommers, Phys. Rev. Lett. 66, 2697 (1991) [erratum: Phys. Rev. Lett. 69, 2738 (1992)].

[7] R. J. Protheroe and D. Kazanas, Astrophys. J. 265620 (1983).

[8] Y. Inoue, D. Khangulyan, S. Inoue and A. Doi, Astrophys. J. 880, 40 (2019) [arXiv:1904.00554].

[9] K. Murase, S. S. Kimura and P. Meszaros, Phys. Rev. Lett. 125, 011101 (2020) [arXiv: 1904.04226].

[10] Y. Inoue, D. Khangulyan and A. Doi, Astrophys. J. Lett. 891, L33 (2020) [arXiv:1909.02239].

[11] A. Kheirandish, K. Murase and S. S. Kimura, [arXiv:2102.04475].

[12] A. L. Müller and G. E. Romero, Astron. Astrophys. 636, A92 (2020) [arXiv:2003.12438].

[13] R. F. Mushotzky, Astrophys. J. 256, 92 (1982).

[14] T. J. Turner and K. A. Pounds, Mon. Not. Roy. Astron. Soc. 240, 833 (1989).

[15] F. W. Stecker, Phys. Rev. Lett. 21, 1016 (1968).

[16] M. Guainazzi, S. Molendi, P. Vignati, G. Matt and K. Iwasawa, New Astron. 5, 235 (2000) [arXiv:astro-ph/9910193].

[17] A. J. Young, A. S. Wilson and P. L. Shopbell, Astrophys. J. 556, 6 (2001) [arXiv:astro$\mathrm{ph} / 0104027]$.

[18] D. A. Smith and A. S. Wilson, Astrophys. J. 591, 138 (2003) [arXiv:astro-ph/0303387].

[19] J. F. Gallimore, S. A. Baum and C. P. O’Dea, Astrophys. J. 613, 794 (2004) [arXiv:astro$\mathrm{ph} / 0406062]$.

[20] F. E. Bauer et al., Astrophys. J. 812, 116 (2015) [arXiv:1411.0670]. 
[21] A. Marinucci et al., Mon. Not. Roy. Astron. Soc. 456, L94 (2016) [arXiv:1511.03503].

[22] G. Piccinotti, R. F. Mushotzky, E. A. Boldt, S. S. Holt, F. E. Marshall, P. J. Serlemitsos and R. A. Shafer, Astrophys. J. 253, 485 (1982).

[23] V. Singh, P. Shastri and G. Risaliti, Astron. Astrophys. 532, A84 (2011) [arXiv:1101.0252].

[24] R. B. Tully, Nearby galaxies catalog, (Cambridge University Press, 1988).

[25] M. G. Aartsen et al. [IceCube, Fermi-LAT, MAGIC, AGILE, ASAS-SN, HAWC, H.E.S.S., INTEGRAL, Kanata, Kiso, Kapteyn, Liverpool Telescope, Subaru, Swift NuSTAR, VERITAS and VLA/17B-403], Science 361, no.6398, eaat1378 (2018) [arXiv:1807.08816].

[26] D. Kazanas and D. C. Ellison, Astrophys. J. 304, 178 (1986).

[27] A. P. Szabo and R. J. Protheroe, Astropart. Phys. 2, 375 (1994) [arXiv:astro-ph/9405020].

[28] S. R. Kelner, F. A. Aharonian and V. V. Bugayov, Phys. Rev. D 74, 034018 (2006) [erratum: Phys. Rev. D 79, 039901 (2009)] [arXiv:astro-ph/0606058].

[29] G. M. Frichter, T. K. Gaisser and T. Stanev, Phys. Rev. D 56, 3135 (1997) [arXiv:astro$\mathrm{ph} / 9704061]$.

[30] F. W. Stecker, Phys. Rev. 180, 1264 (1969).

[31] L. A. Anchordoqui, Phys. Rept. 801, 1 (2019) [arXiv:1807.09645].

[32] N. I. Shakura and R. A. Sunyaev, Astron. Astrophys. 24, 337 (1973).

[33] R. J. Protheroe and A. P. Szabo, Phys. Rev. Lett. 69, 2885 (1992).

[34] F. W. Stecker, Astrophys. J. 228, 919 (1979).

[35] S. R. Kelner and F. A. Aharonian, Phys. Rev. D 78, 034013 (2008) [erratum: Phys. Rev. D 82, 099901 (2010)] [arXiv:0803.0688].

[36] E. Roulet and F. Vissani, [arXiv:2011.12769].

[37] P. Padovani and C. M. Urry, Astrophys. J. 356, 75 (1990).

[38] F. W. Stecker, Phys. Rev. D 72, 107301 (2005) [arXiv:astro-ph/0510537].

[39] F. W. Stecker, Phys. Rev. D 88, 047301 (2013) [arXiv:1305.7404]. 